\title{
Segment Boundary Detection via Class Entropy Measurements in Connectionist Phoneme Recognition *
}

\author{
Giampiero Salvi \\ $K T H$, \\ School of Computer Science and Communication, \\ Speech, Music and Hearing, \\ Stockholm, Sweden
}

\begin{abstract}
This article investigates the possibility to use the class entropy of the output of a connectionist phoneme recogniser to predict time boundaries between phonetic classes. The rationale is that the value of the entropy should increase in proximity of a transition between two segments that are well modelled (known) by the recognition network since it is a measure of uncertainty. The advantage of this measure is its simplicity as the posterior probabilities of each class are available in connectionist phoneme recognition.

The entropy and a number of measures based on differentiation of the entropy are used in isolation and in combination. The decision methods for predicting the boundaries range from simple thresholds to neural network based procedure.

The different methods are compared with respect to their precision, measured in terms of the ratio between the number $C$ of predicted boundaries within 10 or 20 msec of the reference and the total number of predicted boundaries, and recall, measured as the ratio between $C$ and the total number of reference boundaries.
\end{abstract}

Key words: boundary detection, entropy, connectionist phoneme recognition PACS: 43.72.Bs, 43.72.Ne, 43.60.Np

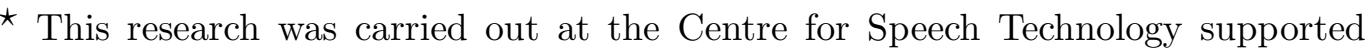
by Vinnova (The Swedish Agency for Innovation Systems), KTH and participating Swedish companies and organisations.

Email address: giampi@kth.se (Giampiero Salvi).

URL: http://www.csc.kth.se/ giampi (Giampiero Salvi).
} 


\section{Introduction}

In a number of speech analysis and recognition applications it is essential to estimate the boundaries between phonetic segments. In landmark (Liu, 1996) and segment based (Glass, 2003; Ostendorf and Veilleux, 1994) recognition this information is used to find suitable locations in the speech signal where to measure the features that constitute an acoustic observation for further analysis.

In the Synface system (Karlsson et al., 2003) a phoneme recogniser based on a hybrid of neural networks and hidden Markov models is used to derive the lip movements of an avatar (Beskow, 2004). The avatar is used to provide lip reading support for hearing impaired people communicating via telephone. In this application both the recognition accuracy and the precision in predicting the segment boundaries are important to synchronise the lip movements to the acoustic signal.

The task of detecting phonetic boundaries is related, but not equivalent, to that of aligning the speech signal to a reference transcription (Hosom, 2002). In alignment, the number of phonetic boundaries is determined by a reference transcription, in detection it is based exclusively on measures obtained from the speech signal, adding a degree of freedom to the problem.

The use of connectionist models for phoneme classification suggests the use of entropy measures based on the posterior probabilities of the phonetic classes given an observation. These are calculated by the neural network for each speech frame. The entropy of the posterior probabilities has been shown to be a good confidence measure on the frame-by-frame classification results (Williams and Renals, 1999; Salvi, 2006a).

Here we focus on the time variation of the entropy and observe that, as a measure of uncertainty, it should present local maxima at the transitions between consecutive segments that are well modelled by the recognition network. This can be explained by noting that, in proximity to a boundary, a number of overlapping frames, and therefore the resulting acoustic features vectors, contain characteristics of both phonetic classes. The recognition network is likely to estimate, in these cases, class probabilities with higher uncertainty.

In Salvi (2006b) it was shown that the value of the entropy at frame $n$ and its first and second derivatives are dependent to the distance of the frame from a phonetic boundary. In this paper the idea is further developed and differential measures of the entropy are used as predictors of phonetic boundaries.

The different methods are compared counting the number $C$ of predicted boundaries that fall within a number of milliseconds from the reference bound- 
aries. Two measures of performance are considered: precision relates $C$ to the total number of predicted boundaries, while recall relates $C$ to the total number of reference boundaries.

The rest of the paper is organised as follows: Section 2 describes the recogniser and the data used in the experiments. Section 3 explains the method and the measures analysed in the experiments. Section 4 presents the results. Finally Section 5 discusses the results and Section 6 concludes the paper.

\section{The Framework}

\subsection{The Recogniser}

The Synface recogniser is a hybrid of recurrent neural networks (RNNs), and hidden Markov models (HMMs). The recogniser is described in detail in Salvi $(2003,2006 a)$, here we mention only the aspects that are relevant to this study.

The input layer of the RNN contains thirteen units that represent the Mel frequency cepstral coefficients $C_{0}, \ldots, C_{12}$.

The activities $a_{i}[n]$ of the output layer give an estimate of the posterior probabilities $P\left(x_{i} \mid O[n]\right)$ of each of the $N=N_{\mathrm{p}}+N_{\mathrm{n}}$ acoustic classes $x_{i}$, given the observation $O[n]$. The acoustic classes include $N_{\mathrm{p}}$ phonetic classes and $N_{\mathrm{n}}$ noise and silence classes. The total number of acoustic classes is 50 for Swedish, with 46 phonemes and 4 kinds of noise/silence.

The posterior probabilities are fed into a Viterbi-like decoder where the lookahead length can be varied. The recognition network specified by a Markov chain defines a loop of phonemes, where every phoneme is represented by a three state left-to-right HMM.

\subsection{Data}

The recogniser was trained on the Swedish SpeechDat database (Elenius, 2000). The database has been divided into a training, a validation and a test set, with 33062, 500 and 4150 utterances, respectively. The validation set was used during training of the recogniser to optimise a number of factors, e.g. the number of units in the hidden layer, the type and number of connections between the layers, and a number of parameters related to the training procedure. Mel frequency cepstral coefficients were computed at every $10 \mathrm{msec}$. 

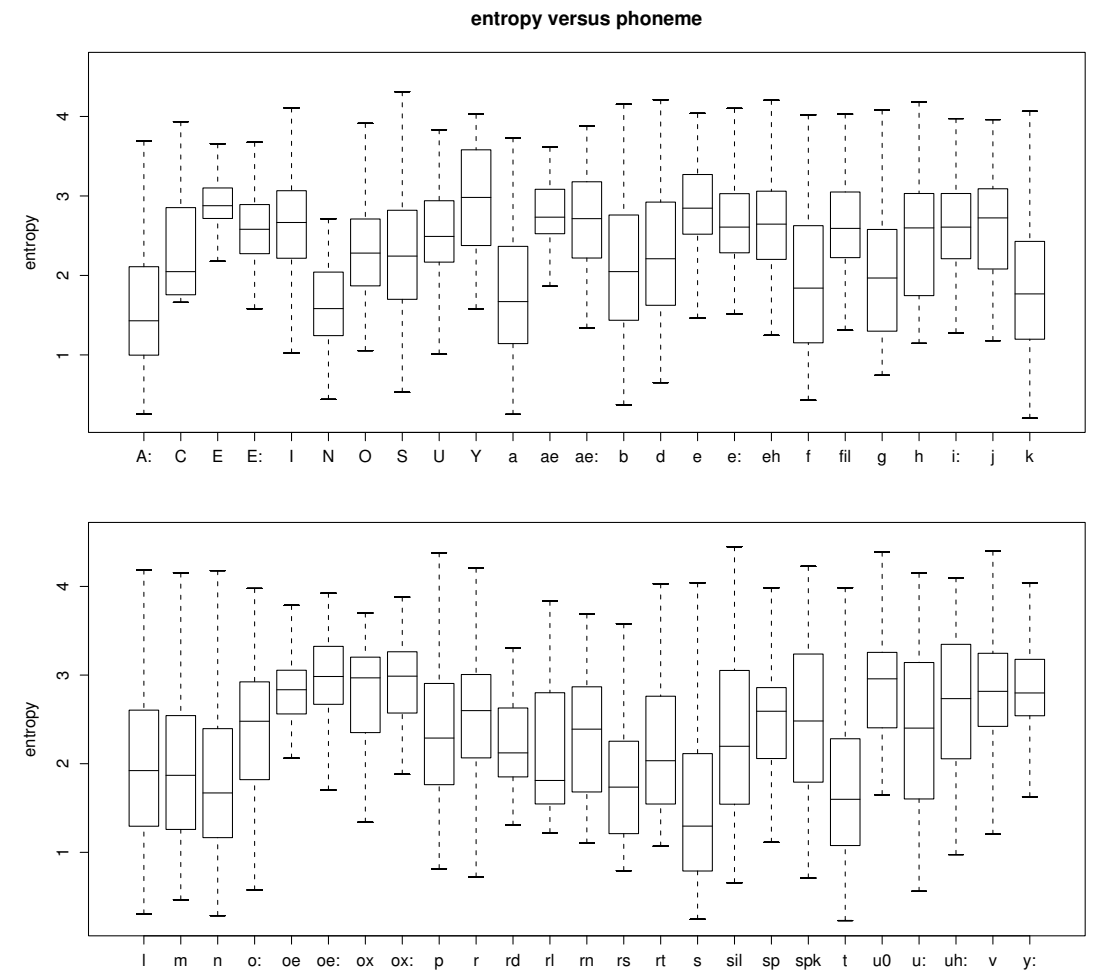

Figure 1. Box plot of the frame entropy for each phonemic class. The maximum entropy is $\log _{2} 50=5.64$ bits. The SAMPA phonetic symbols (Gibbon et al., 1997) are used with a few exceptions (Lindberg et al., 2000): $\} \rightarrow$ uh, $2 \rightarrow$ ox,$\{\rightarrow$ ae, $9 \rightarrow$ oe, $\mathbb{C} \rightarrow$ eh

The phonetic transcriptions used during training of the recogniser, have been obtained with forced alignment. These transcriptions are sufficiently accurate for the task of training the neural networks, but cannot be safely adopted as a reference when the task is to evaluate procedures for boundary detection. In order to obtain a more reliable reference, a number of utterances, randomly chosen from the test material, have been manually transcribed. These utterances are used in the experiments described in the paper. Given the time consuming process of manually transcribing speech material, only 80 test utterances were used. In our opinion, the resulting total number of boundaries (2816), constitutes a good statistical sample for the experiments of this paper.

\section{Method}

As introduced in Section 2 the activities $a_{i}[n]$ of the output nodes of the phoneme recognition network give an estimate of the posterior probabilities $P\left(x_{i} \mid O[n]\right), i \in[1, N]$ of the acoustic class $x_{i}$ given the observation $O[n]$ at 


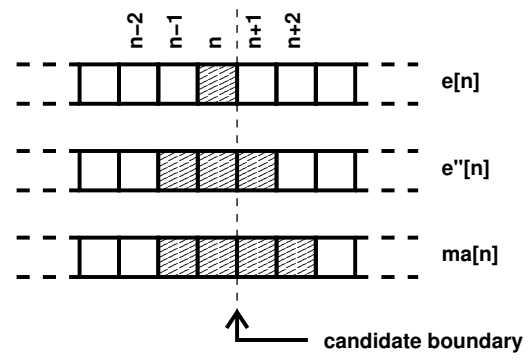

Figure 2. Illustration of the time span of each measure with respect to the candidate boundary

frame $n$. The class entropy of the frame is defined as

$$
e[n]=-\sum_{i=1}^{N} a_{i}[n] \log _{2} a_{i}[n]
$$

Figure 1 shows the box plot of the entropy in Equation 1 for each of the 50 phonetic classes (including noise). The higher bound to the entropy is $\log _{2} 50=5.64$ bits.

The candidates for the boundaries are estimated by searching for local maxima in the entropy function. This is a problem of peak picking, in relatively unusual conditions. Most of the literature on peak picking (e.g. Li et al., 1995; Andreev et al., 2003), in fact, deals with the problem of estimating the peaks (local maxima) of a process affected by noise. Smoothing methods are employed to eliminate the effect of the noise fluctuations, preserving the shape of the "clean" process. Once the smoothing is applied, the local maxima are obtained by simply imposing that the first derivative of the measure, with respect to time, is nearly 0 , when the second derivative is negative. This is possible when the function of time is sampled at a sufficiently high rate compared with its time fluctuations.

In our case, the entropy is computed on a number of probability estimators that, in turn, operate on a frame of speech samples. The entropy is thus a smooth function of time, and the effects of additive noise are limited. However, the main problem in our application is that this function is sampled at a low rate, compared to its fluctuations. One consequence is that the first derivative looses its property of vanishing in proximity to a maximum (or minimum or inflection point) and cannot be used in isolation to determine the positions of the maxima.

A number of methods have been investigated in order to find the local maxima in the entropy function. The simplest method, referred to as $\mathbf{e}[\mathbf{n}]$ in the following, applies a threshold on the entropy function. As illustrated in Figure 1, the entropy is not only dependent on the proximity to a boundary as postulated up to now, but also on the phonetic segment. This limits the effectiveness of 
Table 1

Summary of the boundary detection methods, The difference between the decision

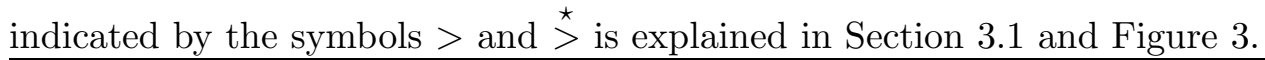

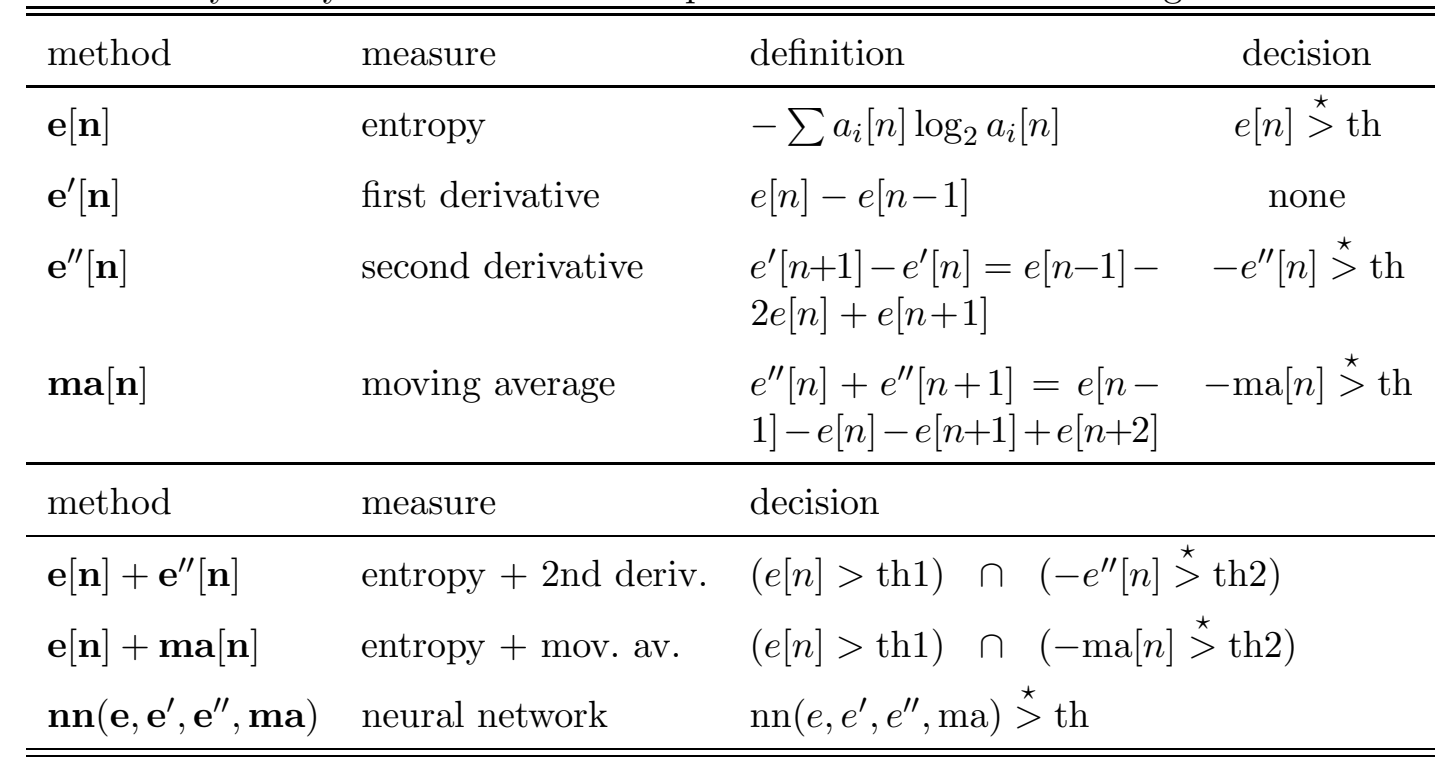

this simple method, as it is hard to find a single value for the threshold that would work in general.

A solution to this is the use of differential measures. As already mentioned, the first derivative of the entropy in these conditions bears limited information about the position of the boundaries. The second derivative defined as

$$
e^{\prime \prime}[n]=e^{\prime}[n+1]-e^{\prime}[n]=e[n-1]-2 e[n]+e[n+1]
$$

on the contrary, is a good indication of local maxima, as low values correspond to regions where the function $e[n]$ is concave, and, given the low sampling rate, these regions are likely to contain local maxima.

As illustrated in Figure 2 each sample of the entropy function refers to a segment (frame), while the boundaries are between two adjacent frames. It is therefore desirable to base the decision on whether there is a boundary between frame $n$ and frame $n+1$ on a measure that is symmetric with respect to these two frames. One way of doing this is to average the second derivative over two adjacent frames (moving average of the second order) that, besides a constant factor, is:

$$
\operatorname{ma}[n]=e^{\prime \prime}[n]+e^{\prime \prime}[n+1]=e[n-1]-e[n]-e[n+1]+e[n+2]
$$

Table 1 gives a summary of the methods used in our experiments. As shown in the table the boundaries were predicted by setting a threshold on each of the above measures in isolation, or by combining them. Two ways of combining 
the measures were considered. The first way is motivated by the attempt to reduce the insertions of boundaries obtained with the second derivative and with the moving average methods. The absolute value of the entropy is used in this case to eliminate those cases in which local maxima are detected in regions of globally low entropy (spurious maxima). The second combination method makes use of a time delayed neural network with the regression task to estimate a function of proximity to a boundary. In this case all the measures are given in input to the network in order to let the training procedure find an optimal combination. As the target values to the neural network should be bounded between known limits (e.g. 0 and 1), it was necessary to map the distance $d(f, b) \in[0, \infty)$ between frame $f$ and boundary $b$ to the interval $(0,1)$. A convenient way of doing this is by using the exponential function. The resulting measure, defined as:

$$
p(f, b)=e^{-d(f, b)}
$$

will be called proximity in the following. A frame adjacent to a boundary has distance $d=0$ and proximity $p=1$. As the distance $d \rightarrow \infty$ the proximity $p \rightarrow 0$. The neural network is composed by an input, a hidden and an output layer of respectively 4, 11 and 1 units. The layers are fully connected with time-delayed arcs. The estimate of the function $p(f, b)$ by the neural network will be called $\mathbf{n n}\left(\mathbf{e}, \mathbf{e}^{\prime}, \mathbf{e}^{\prime \prime}, \mathbf{m a}\right)$ in the following.

\subsection{Decision Method}

The decision procedure indicated by the relation $m>$ th simply finds the regions in which the measured $m$ exceeds the threshold th.

The decision procedure indicated by the relation $m>^{\star}$ th, instead, picks only one boundary candidate for each region of contiguous frames for which the measure exceeds the threshold. This avoids detecting adjacent boundary candidates, but leads to boundary deletions in case two or more local maxima exist in the same region as illustrated in Figure 3.

In case of combined measures, the regions in which the entropy $e[n]$ exceeds the threshold are first found with method $>$, then, within these regions method

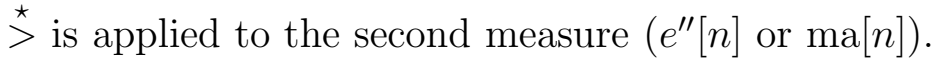




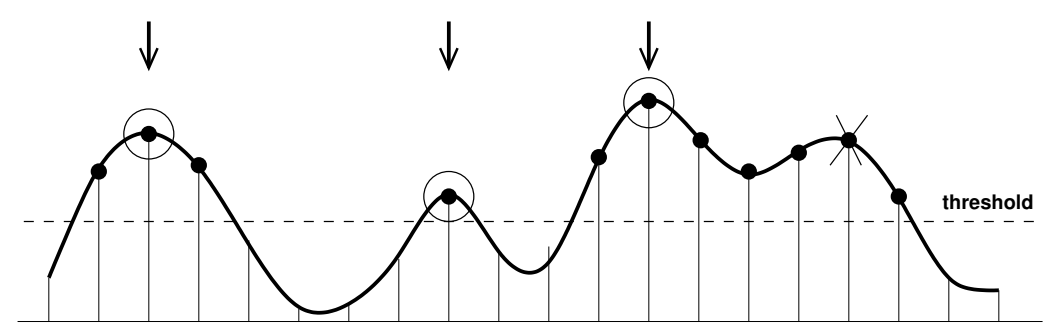

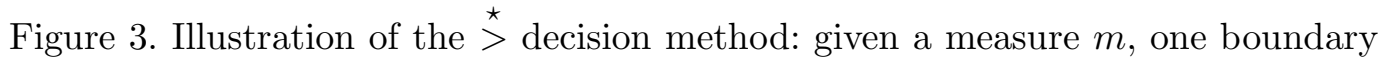
candidate is selected for each region of contiguous frames for which $m>$ th. The candidate is the global maximum for $m$ in that region. The vertical arrows indicate the resulting boundaries, the cross corresponds to a possible deletion.

\subsection{Threshold}

The value of the threshold in the decisions above can be used to find a trade-off between number of boundary insertions and deletions. The range of suitable values for the thresholds depends on the measurements they are applied to. In the following, all threshold values are relative to the global statistics for each measured variable. Calling $\mu_{i}$ and $\sigma_{i}$ respectively the mean and standard deviation for the $i$ th variable, the value for the corresponding threshold ranges from $\mu_{i}-\sigma_{i}$ to $\mu_{i}+2 \sigma_{i}$ and will be indicated with relative values from -1.0 to 2.0 .

When multiple measures are combined, an optimisation is performed to determine the best combination of values.

\subsection{Baseline}

As the phoneme recognition network estimates the posterior probabilities for each phonetic class given the observation (frame), its output can be used directly as a classifier of each input frame in a maximum a posteriori sense. The phonetic boundaries could be therefore detected by simply observing the changes in classification from frame to frame. This method constitutes the baseline of our investigation.

\subsection{Level of Chance}

To determine the advantage in using any of the above methods, it is interesting to measure the results obtained by randomly assigning $F$ boundaries to the $M$ possible positions, where $M+1$ is the number of frames in the utterance. We call this measure level of chance. 
First we notice that, disregarding the effect of the beginning and end of each utterance, if we have $B$ non contiguous reference boundaries in $M$ positions, the probability that a randomly chosen position $f$ is within one frame from any of the $B$ boundaries $b_{i}$ is

$$
\sum_{i=1}^{B} p\left(d\left(f, b_{i}\right) \leq 1\right) \approx \frac{3 B}{M}
$$

If we consider $F$ randomly selected positions and $B \ll M$ and $F \ll M$, the above approximation is still valid, and the level of chance, that is the ratio of positions $f_{j}$ within one frame of any reference boundary is simply $\frac{3 B}{M}$, independently of $F$. Similarly the level of chance will be $\frac{5 B}{M}$ if we consider boundaries with at most $20 \mathrm{msec}$ distance from the reference. In the test material, the percentage of reference boundary per frame is $B / M \simeq 0.114$ which gives a theoretical level of chance of $34.2 \%$ for $10 \mathrm{msec}$ and $57.0 \%$ for 20 msec.

This value was verified with Monte Carlo simulations where the detected boundaries were obtained by sampling without repetition the available $M$ positions. The size of the sample $F$ was a predefined ratio of the number $B$ of boundaries in the reference transcription for each utterance. The ratio was varied in a way to simulate the effect of varying the threshold (and thus the number of insertions and deletions) in the methods described above. For each ratio, the simulation was repeated 50 times over which the results were averaged.

\subsection{Forced Alignment}

Another reference for the performance of the methods considered in this paper are the results obtained with forced alignment using the recognition models, and the original transcriptions. It should be clear in this case that the problem of detecting phoneme boundaries is completely different from the problem of aligning an already known sequence of phonemes to the acoustic observations. In the first case, in fact, no a priori information on the correct sequence of phonemes (and as a consequence of the number of phonetic boundaries) is given, introducing a number of degrees of freedom that make the problem more challenging. The results are thus not directly comparable, but the forced alignment results could be interpreted as a higher bound to the boundary detection methods. 


\subsection{Evaluation}

Two evaluation measures are used to compare the different methods. In both cases the number of correctly detected boundaries $C$ is first computed. A correctly detected boundary is defined as a boundary that lies within a number of milliseconds from a reference boundary.

Two definitions of the time limit have been used in this paper following the literature: Liu (1996) uses $10 \mathrm{msec}$ as a limit of correctness, while (e.g. Hosom, 2002) uses 20 msec. Most of the analysis are performed with the $10 \mathrm{msec}$ limit, while the final results are presented for both limits.

Calling $D$ the total number of detected boundaries and $T$ the total number of reference (transcription) boundaries, the two measures of performance are: precision $P$ computed as $C / D \%$, and recall $R$ computed as $C / T \%$. Precision counts the percentage of correctly predicted boundaries with respect of the total number of predicted boundaries. Recall counts the percentage of correctly predicted boundaries with respect of the total number of reference boundaries.

In the cases when an optimisation is performed, the above two measures are combined in a single criterion that is defined as the Euclidean distance between the point defined as $(P, R)$, and the point $(100 \%, 100 \%)$, that is:

$$
\text { crit }=\sqrt{(P-100)^{2}+(R-100)^{2}}
$$

The optimisation tends to minimise this criterion. Note that this criterion gives the same weight to precision and recall. In some applications it may be more convenient to prioritise one of the two.

The procedure, described above, for computing the number of correctly detected boundaries overlooks insertions. If two or more detected boundaries are in the proximity of the same reference boundary, they are all considered to be correct. This does not constitute a limitation in most of the cases considered

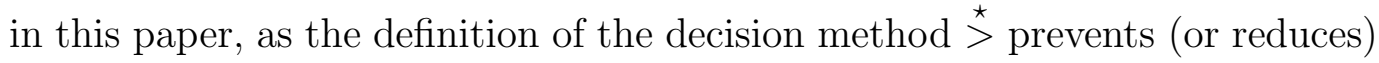
detecting boundaries that are too close in time. The baseline method, however, does not have such a constraint, and can in principle predict a phonetic boundary for each time step. In this case, the number $C$ of correct boundaries could exceed the number of reference boundaries $T$. This will affect both the measure of precision and recall that can eventually exceed $100 \%$. 

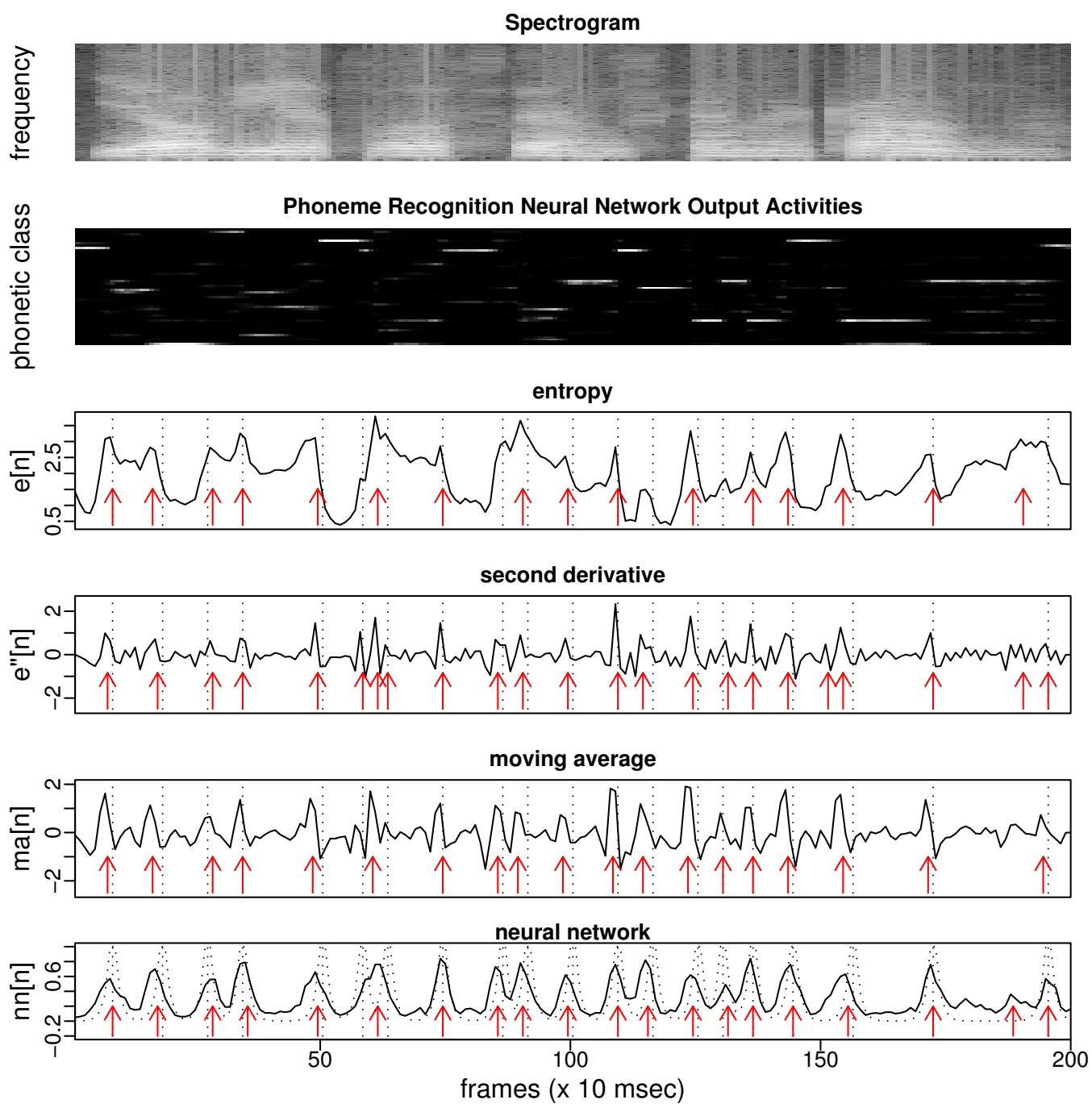

Figure 4. Example of test utterance containing the phrase "jag vet vad svenskarna kan" ([jA:ve:tvA:svenskarnakan]. The last four plots show different measures described in the text (continuous line), the reference boundaries (dotted lines) and the predicted boundaries (arrows). The last plot also include the proximity function (dotted line) used as target for the neural network training.

\section{Results}

\subsection{Example}

Figure 4 illustrates some of the measures defined in Section 3 for one test utterance containing the phrase "jag vet vad svenskarna kan" ([jA:ve:tvA:svenskarnakan]).

The top plot shows the spectrogram of the signal, the second plot the output activities $a_{i}[n]$ of the phoneme recognition network. The last four plots show four of the measures defined in Table 1 . The vertical dotted lines are the 
reference boundaries while the arrows are the predicted boundaries when the threshold was chosen in order to obtain a total number of predicted boundaries closest to the total number of reference boundaries.

The first measure is the entropy $\mathbf{e}[\mathbf{n}]$. The boundary detection and alignment is acceptable, but a few deletions can be noticed. The second measure is the second derivative of the entropy $\mathbf{e}^{\prime \prime}[\mathbf{n}]$. This shows an improvement compared to $\mathbf{e}[\mathbf{n}]$ in many cases as the number of deletions is reduced, however it introduces a number of insertions.

The third measure is the moving average of the second derivative of the entropy $\mathbf{m a}[\mathbf{n}]$. Compared to the second derivative this eliminates some insertions.

The last measure is the output of the time delayed neural network $\mathbf{n n}\left(\mathbf{e}, \mathbf{e}^{\prime}, \mathbf{e}^{\prime \prime}, \mathbf{m a}\right)$. In this case the bound proximity function (Equation 2) is shown as well as the reference boundaries in dotted lines. The first observation is that the neural network does a discrete job in predicting the time evolution of the proximity function. This in spite of the fact that the extreme values 0 and 1 are never reached. The second observation is that despite one deletion on the sixth boundary and one insertion before the last, this measure performs best in the example.

\subsection{Combined Methods: Threshold optimisation}

Figure 5 shows the optimisation of the two thresholds th1 and th2 for the two combined methods: $\mathbf{e}[\mathbf{n}]+\mathbf{e}^{\prime \prime}[\mathbf{n}]$ and $\mathbf{e}[\mathbf{n}]+\mathbf{m a}[\mathbf{n}]$. Precision and recall are shown on the left plots, while the criterion in Equation 3 is shown in the right plots. As expected, precision decreases when recall increases. The precision increases overall when both thresholds are increased, because the methods become more selective. Both the precision and recall surfaces have a bell shape in the direction of th2 with a minimum (maximum) around th $2=0$.

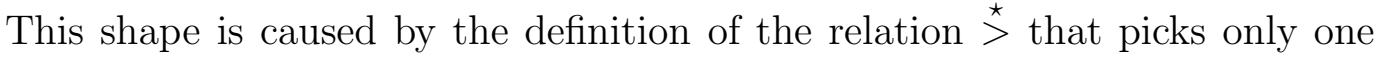
candidate for each region of contiguous measures that exceed the threshold. Increasing th2 the regions become fewer and smaller, and, when th2 is higher than the maximum value for the measure, no region is produced. Decreasing th2 the regions become larger. Below a certain value of th2 the regions become fewer again and, for values of th2 lower than the measure's minimum, there is only one region (and thus one predicted boundary) for each utterance.

The values of th1 are varied between -2 and 2 (in relative terms). The lower the value of th1, the higher the number of samples that satisfy the conditions $e[n]>t h 1$, the more the two combined methods $\mathbf{e}[\mathbf{n}]+\mathbf{e}^{\prime \prime}[\mathbf{n}]$ and $\mathbf{e}[\mathbf{n}]+\mathbf{m a}[\mathbf{n}]$ become similar to the simpler methods $\mathbf{e}^{\prime \prime}[\mathbf{n}]$ and $\mathbf{m a}[\mathbf{n}]$. 


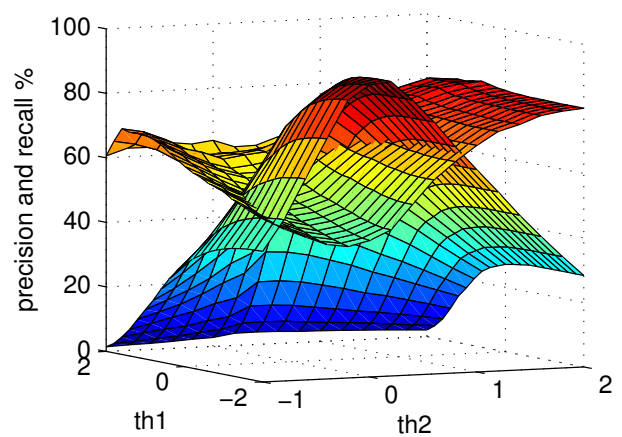

(a)

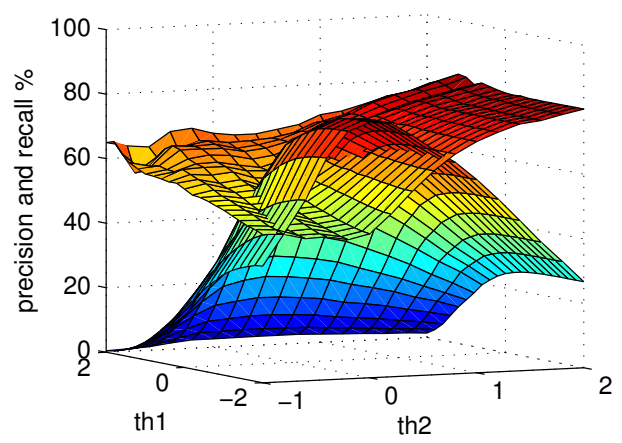

(c)

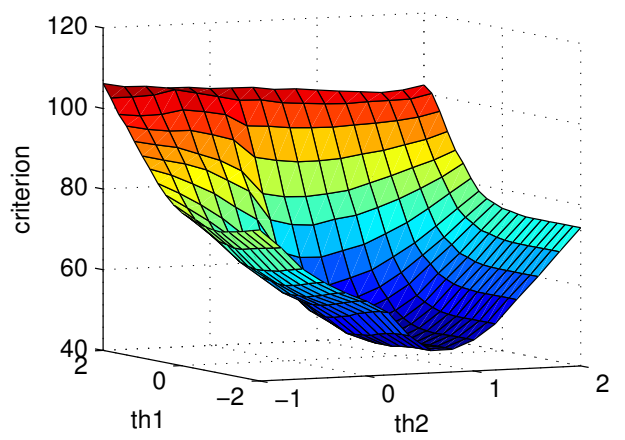

(b)

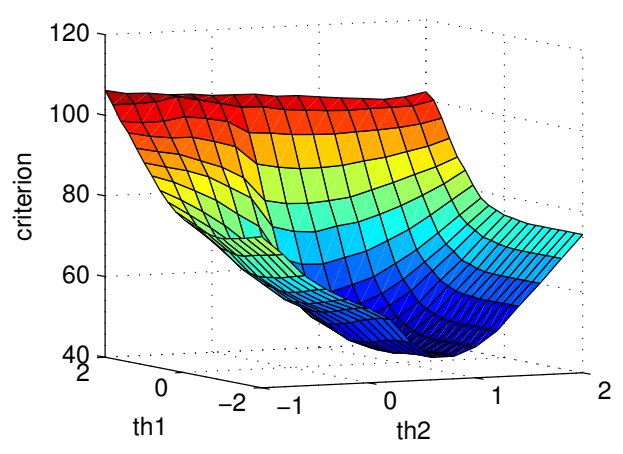

(d)

Figure 5. Threshold optimisation for method $\mathbf{e}[\mathbf{n}]+\mathbf{e}^{\prime \prime}[\mathbf{n}]$ (a,b) and method $\mathbf{e}[\mathbf{n}]+\mathbf{m a}[\mathbf{n}](\mathrm{c}, \mathrm{d})$. Plots (a) and (c) show the recall $R$ (surface with the more pronounced maximum) and the precision $P$ as a function of the two thresholds th1 and th2. The $100 \%$ plane is also shown in the plots. Plots (b) and (d) show the optimality criterion crit as a function of th1 and th2.

Figure 6 shows the results of the optimisation of the value of th1 with respect to th2, obtained by minimising the criterion in Equation 3. The top plot shows the best value of th1 as a function of th2 for the two methods $\mathbf{e}[\mathbf{n}]+\mathbf{e}^{\prime \prime}[\mathbf{n}]$ and $\mathbf{e}[\mathbf{n}]+\mathbf{m a}[\mathbf{n}]$, and the bottom plot shows the resulting values for the criterion.

\subsection{Result Summary}

Figure 7 presents a summary of the results obtained with all methods in Table 1 by varying the value of the relative threshold. In case of combined measures, the optimal value of the threshold th1, obtained as described in the previous section, is used for each value of th2. All results in the figure refer to $10 \mathrm{msec}$ correct boundaries. The first two plots show the precision $(10 \mathrm{msec})$ and the recall $(10 \mathrm{msec})$ as functions of the relative threshold.

In the third plot at the bottom of Figure 7, the relative threshold variable is 

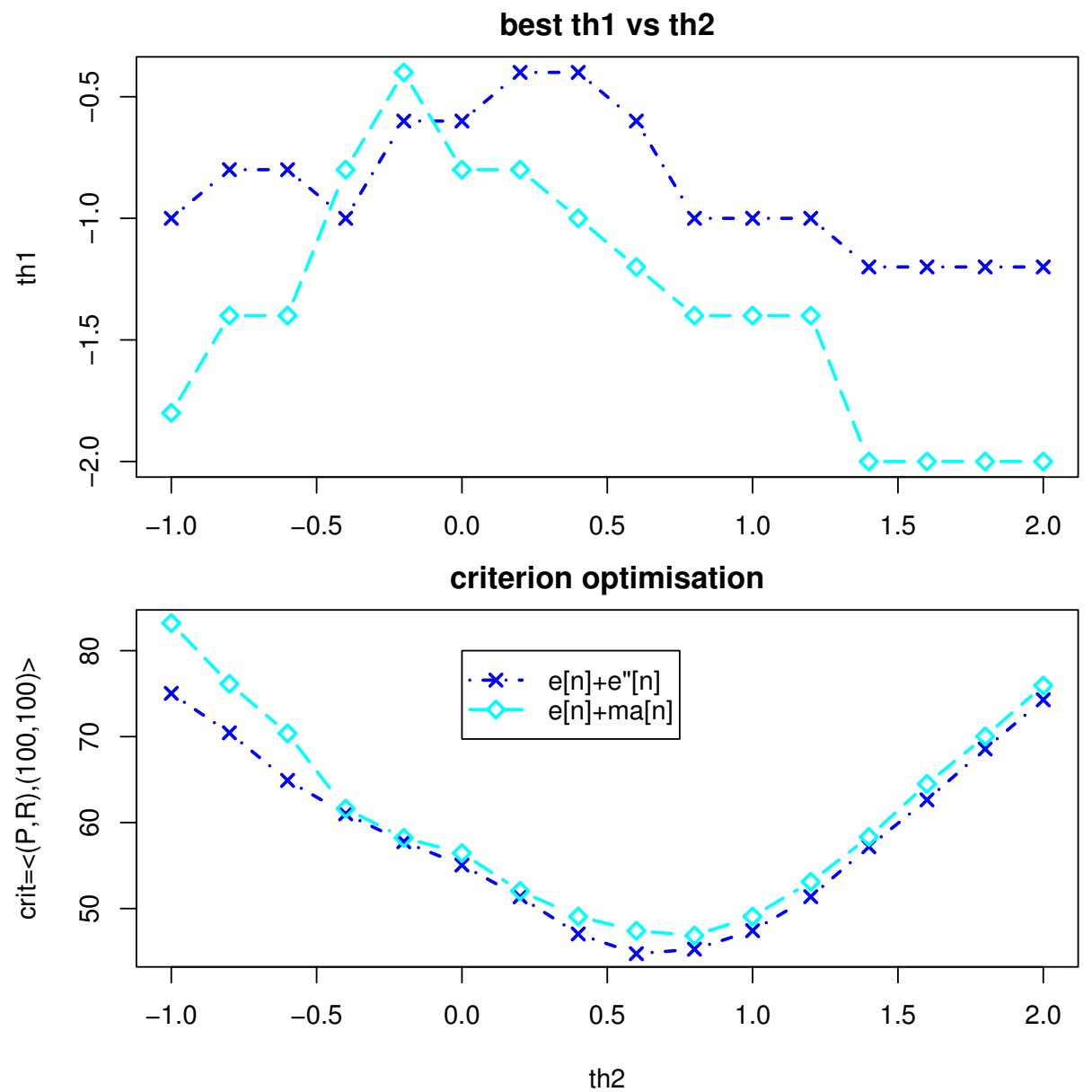

Figure 6. Results of the threshold optimisation for methods $\mathbf{e}[\mathbf{n}]+\mathbf{e}^{\prime \prime}[\mathbf{n}]$ and $\mathbf{e}[\mathbf{n}]+\mathbf{m a}[\mathbf{n}]$. Above: optimal relative value of th1 in function of th2. Below: corresponding value of the criterion in Equation 3

eliminated and precision is plotted in function of recall. The dependency with the relative threshold is implicitly shown in this plot by the trajectories of each group of result points. In this last plot the baseline result is reported as well as the level of chance for different values of recall. The precision of the level of chance, that was obtained with the Monte Carlo simulations, is in good agreement with the theoretical value found in Section 3.4, with values that range from $34.6 \%$ to $34.9 \%$. The recall varies between $1 \%$ and $86.8 \%$ when the total number of predicted boundaries (that is a factor in the simulations) is varied between $5 \%$ and $250 \%$ of the total number of reference boundaries.

The recall for the baseline condition exceeds 100\%. As already noticed in Section 3.6, this is due to the way the number of correctly detected boundaries is computed. Given this limitation, the value for the optimality criterion is unreliable and should not be used to compare this conditions to the others. The precision is, however, an interesting figure to determine the performance of this method. 

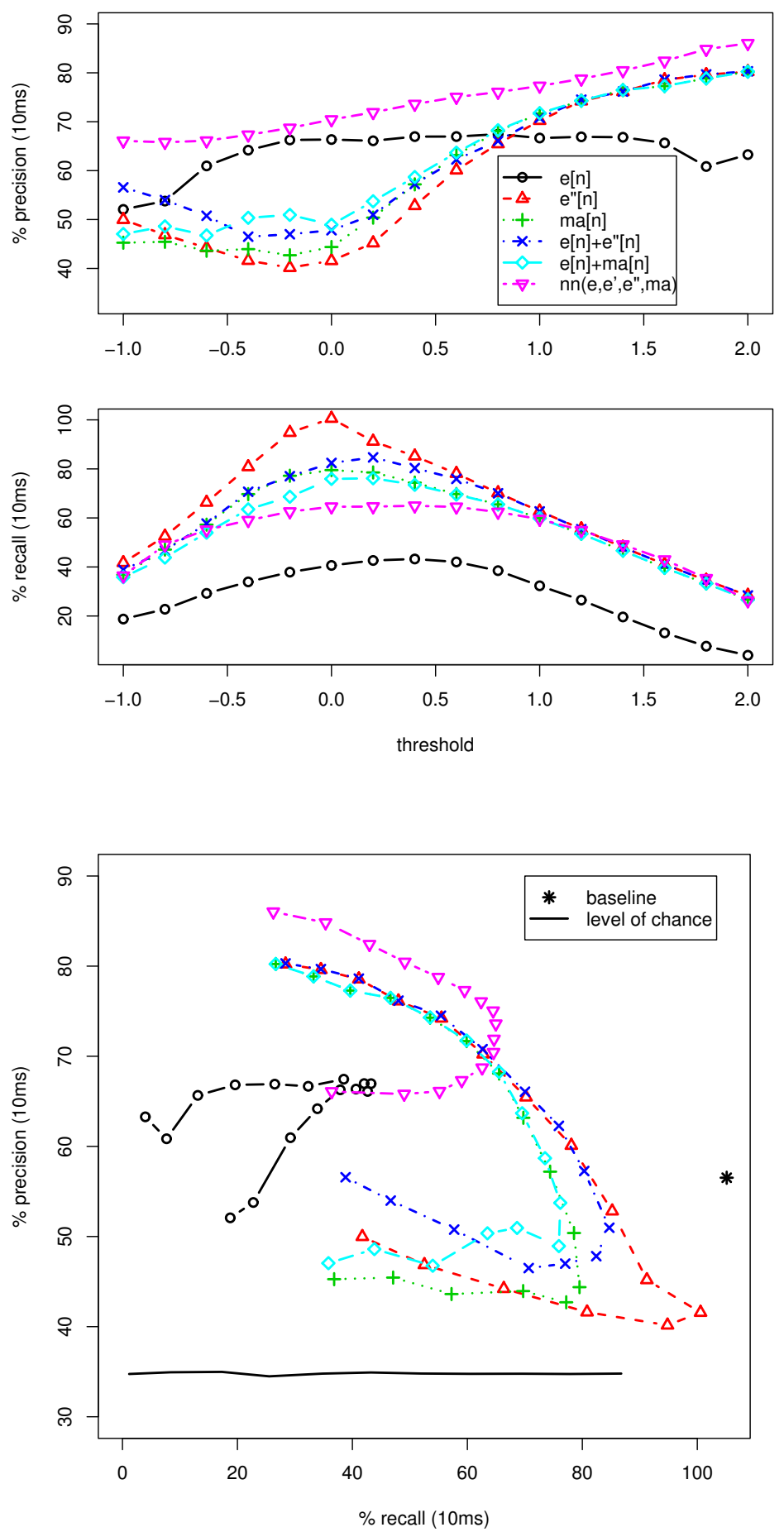

Figure 7. Summary of the results for varying relative thresholds. From the top: the precision and the recall plotted against the relative threshold. The bottom figure plots the precision versus the recall when the threshold variable is eliminated. In this plot the baseline result and the level of chance, obtained with Monte Carlo simulations, are also reported. 
Table 2

Summary of the best results. The values correspond to correctness within 10 (20) msec of the reference boundaries, $\left.{ }^{*}\right)$ the recall, and therefore the criterion, are in this case arbitrary, as the total number of predicted boundaries is a factor that does not change the precision. The $100 \%$ recall, and the corresponding criterion are reported for comparison with the other conditions. ${ }^{* *}$ ) the reason why the recall can be $>100 \%$ is explained in the text.

\begin{tabular}{lccc}
\hline \hline condition & precision \% & recall \% & criterion \\
\hline level of chance & $34.8(55.0)$ & $100(100)^{*}$ & $65.2(45.0)^{*}$ \\
baseline & $56.5(74.3)$ & $105(138)^{* *}$ & $43.8(46.0)$ \\
$\mathbf{e}[\mathbf{n}]$ & $67.0(81.0)$ & $43.3(52.3)$ & $65.7(51.3)$ \\
$\mathbf{e}^{\prime \prime}[\mathbf{n}]$ & $60.1(79.8)$ & $78.1(85.5)$ & $45.5(24.9)$ \\
$\mathbf{m a}[\mathbf{n}]$ & $68.1(78.5)$ & $65.5(86.6)$ & $47.0(25.3)$ \\
$\mathbf{e}[\mathbf{n}]+\mathbf{e}^{\prime \prime}[\mathbf{n}]$ & $62.3(77.6)$ & $75.9(94.6)$ & $44.8(23.0)$ \\
$\mathbf{e}[\mathbf{n}]+\mathbf{m a}[\mathbf{n}]$ & $68.3(79.1)$ & $65.5(86.3)$ & $46.9(25.0)$ \\
$\mathbf{n n}\left(\mathbf{e}, \mathbf{e}^{\prime}, \mathbf{e}^{\prime \prime}, \mathbf{m a}\right)$ & $75.0(86.4)$ & $64.5(76.2)$ & $43.4(27.4)$ \\
forced alignment & $86.8(93.9)$ & $90.8(98.3)$ & $16.1(6.28)$ \\
\hline \hline
\end{tabular}

The $\mathbf{e}[\mathbf{n}]$ condition shows a low dependency of precision with the threshold value. However the entropy alone underestimates the number of boundaries. The best condition with respect to the criterion in Equation 3 (crit=65.7) gives a precision of $67.0 \%$ and a recall of $43.3 \%$.

The $\mathbf{e}^{\prime \prime}[\mathbf{n}]$ and $\mathbf{m a}[\mathbf{n}]$ conditions clearly outperform $\mathbf{e}[\mathbf{n}]$ in terms of the criterion (45.5 and 47.0 respectively). The first method drastically improves the recall $(78.1 \%)$ at the expense of precision $(60.1 \%)$. The second improves both precision $(68.1 \%)$ and recall $(65.5 \%)$, even tough the last to a lesser degree.

The combined measures $\mathbf{e}[\mathbf{n}]+\mathbf{e}^{\prime \prime}[\mathbf{n}]$ and $\mathbf{e}[\mathbf{n}]+\mathbf{m a}[\mathbf{n}]$, as compared to the corresponding alone conditions $\mathbf{e}^{\prime \prime}[\mathbf{n}]$ and $\mathbf{m a}[\mathbf{n}]$, give in general a higher precision at the expenses of a lower recall (see the first and second plot in Figure 7). The performance in terms of the evaluation criterion is only slightly better than the corresponding alone conditions with $\mathrm{crit}=44.8$ and $\mathrm{crit}=46.9$ respectively.

Finally the $\mathbf{n n}\left(\mathbf{e}, \mathbf{e}^{\prime}, \mathbf{e}^{\prime \prime}, \mathbf{m a}\right)$ condition is best in all cases reaching a precision of $75.0 \%$ with a recall of $64.5 \%$ and a criterion value of 43.4 .

Table 2 summarises the best results obtained with the different methods. The main results are given using $10 \mathrm{msec}$ limits of correctness. In the literature about phoneme alignment (e.g. Hosom, 2002) the precision is often measured in terms of percentage of the boundaries falling within $20 \mathrm{msec}$ of the reference. 
To allow for comparison the corresponding results are reported in parenthesis in the table. For the same purpose, the results obtained by forced alignment as described in Section 3.5, are also shown in the table. It is, however, worth reminding that the task considered in this paper is different from the phoneme alignment task as no reference transcription is used in the discussed methods, to find the phonetic boundaries. The results are therefore not directly comparable.

\section{Discussion}

The fundamental limitation of the methods described in this paper is the fact that the time resolution for the boundary detection is one frame (10 $\mathrm{msec})$. More precise boundary placements can be achieved with measures at the speech sample level. This is not a severe limitation for most speech analysis tasks, also considering that the position of phonetic boundaries is often ambiguous.

A second limitation is the evaluation criterion. In fact the computation of number of correctly detected boundaries overlooks insertions near the boundaries. These can cancel out the number of deletions of boundaries, giving values of precision and recall that are misleadingly high. As already discussed in the paper this problem does not emerge in evaluating the methods here exposed, as they intrinsically avoid detecting boundaries that are too close in time. The definition of a distance measure that uses dynamic programming to guarantee that only one detected boundary is assigned to each reference boundary, might help estimate with more precision the amount of insertions and deletions in the results.

The best methods $\left(\mathbf{e}[\mathbf{n}]+\mathbf{m a}[\mathbf{n}]\right.$ and $\left.\mathbf{n n}\left(\mathbf{e}, \mathbf{e}^{\prime}, \mathbf{e}^{\prime \prime}, \mathbf{m a}\right)\right)$ seem to underestimate the number of boundaries in the material. The corresponding deletions, at a visual inspection, seem to correspond to local maxima in $e[n]$ that were not correctly detected, giving space for future improvements. One way to achieve such an improvement is to implement a more sophisticated decision procedure that could eliminate the problems discussed in Section 3.1.

\section{Conclusions}

In this paper we propose the use of the entropy of the posterior probabilities estimated by a neural network for phoneme recognition, as a predictor of phonetic boundaries. 
A number of measures based on time differentiation of the entropy have been used in isolation and in combination. The best method, based on a non-linear combination of the entropy and its derivatives achieves $75.0 \%$ precision and $64.5 \%$ recall with 10 msec limits of correctness and $86.4 \%$ precision and $76.2 \%$ recall with 20 msec limits.

\section{References}

Andreev, V. P., Rejtar, T., Chen, H.-S., Moskovets, E. V., Ivanov, A. R., Karger, B. L., 2003. A universal denoising and peak picking algorithm for lc-ms based on matched filtration in the chromatographic time domain. Analytical Chemistry 75 (22), 6314-6326.

Beskow, J., 2004. Trainable articulatory control models for visual speech synthesis. Journal of Speech Technology 7 (4), 335-349.

Elenius, K., 2000. Experience from collecting two swedish telephone speech databases. International Journal of Speech Technology 3, 119-127.

Gibbon, D., Moore, R., Winski, R. (Eds.), 1997. Handbook of Standards and Resources for Spoken Language Systems. Mouton de Gruyter, Berlin and New York, Ch. SAMPA computer readable phonetic alphabet, Part IV, section $\mathrm{B}$.

Glass, J. R., 2003. A probabilistic framework for segment-based speech recognition. Computer Speech and Language 17, 137-152.

Hosom, J. P., 2002. Automatic phoneme alignment based on acoustic-phonetic modeling. In: International Conference on Spoken Language Processing (ICSLP). Vol. I. pp. 357-360.

Karlsson, I., Faulkner, A., Salvi, G., 2003. SYNFACE - a talking face telephone. In: Proc. Eurospeech. pp. 1297-1300.

Li, C., Zheng, C., Tai, C., Jan. 1995. Detection of ecg characteristic points using wavelet transform. IEEE Transactions on Biomedical Engineering 42 (1), $21-28$.

Lindberg, B., Johansen, F. T., Warakagoda, N., Lehtinen, G., Kačič, Z., Z̆gank, A., Elenius, K., Salvi, G., 2000. A noise robust multilingual reference recogniser based on SpeechDat(II). In: 6th Intern. Conf. on Spoken Language Processing. Vol. III. pp. 370-373.

Liu, S. A., 1996. Landmark detection for distinctive feature-based speech recognition. Journal of Acoustic Society of America 100 (5), 3417-3430.

Ostendorf, M., Veilleux, N., 1994. A hierarchical stochastic model for automatic prediction of prosodic boundary location. Computational Linguistics 20 (1), 27-54.

Salvi, G., 2003. Truncation error and dynamics in very low latency phonetic recognition. In: ISCA Tutorial and Research Workshop on Non-linear Speech Processing (NOLISP), Le Croisic, France.

Salvi, G., 2006a. Dynamic behaviour of connectionist speech recognition with 
strong latency constraints. Speech Communication 48 (7), 802-818.

Salvi, G., 2006b. Segment boundaries in low latency phonetic recognition. Lecture Notes in Artificial Intelligence 3817, 267-276.

Williams, G., Renals, S., 1999. Confidence measures from local posterior probability estimates. Computer Speech and Language 13 (4), 395-411. 